\title{
EXCESSO DE PESO, NÍVEL DE ATIVIDADE FÍSICA E HÁBITOS ALIMENTARES EM ESCOLARES DA CIDADE DE ANÁPOLIS-GO
}

DRA. PATRÍCIA ESPÍNDOLA MOTA VENÂNCIO

Doutora em Educação Física pela universidade Católica de Brasília (UCB), Professora no curso de Educação Física de Anápolis- UniEVAGÉLICA (Anápolis - Goiás - Brasil)

E-mail: venanciopatricia@yahoo.com.br

\section{DRA. CRISTINA GOMES DE OLIVEIRA TEIXEIRA}

Doutora em Educação Física pela universidade Católica de Brasília (UCB),

Professora no curso de Educação Física de Anápolis-UniEVAGÉLICA

(Anápolis - Goiás - Brasil)

E-mail: cristinagomesteixeiral@hotmail.com

\section{DR. FRANCISCO MARTINS DA SILVA}

Professor do Programa de Pós-graduação em Educação Física da Universidade Católica de Brasília (UCB), Doutorado na Faculdade de Desportos da Universidade do Porto - Portugal, Diretor do Curso de Educação Física da UCB (Brasília - Distrito Federal - Brasil )

E-mail:fmsilva@pos.ucb.br

\begin{abstract}
RESUMO
Este estudo teve como objetivo identificar o excesso de peso, o nível de atividade física e os hábitos alimentares, em escolares com idades entre 7 e 9 anos. $O$ estudo foi de caráter epidemiológico, com delineamento transversal, envolvendo 1982 crianças. Os resultados revelaram que o excesso de peso foi menor nos estudantes do ensino público. Em relação ao nivel de atividade fisica, constatou-se que a maioria dos escolares foi considerada sedentária ou insuficientemente ativa, e que os estudantes das escolas públicas são mais ativos. Concluiu-se que os escolares adotam nível de atividade fisica e hábitos alimentares insuficientes em relação às orientações sugeridas pela literatura científica.
\end{abstract}

PALAVRAS-CHAVE: Excesso de peso; atividade física habitual; hábitos alimentares; escolares. 
Embora a obesidade não seja um fenômeno recente, sua prevalência nunca atingiu as proporções epidêmicas como atualmente se registra (SAVOYE et al., 2007; ORSI; HALE; LYNCH, 20I I). Nos últimos anos, coincidentemente com o que se observa na população adulta, a obesidade tem crescido substancialmente entre crianças e adolescentes (BANKOFF; MOUTINHO, 2002; OLIVEIRA; FISBERG, 2003, SILVA; BALABAN; MOTTA, 2005).

Nos levantamentos realizados em 2008-2009 pelo Instituto Brasileiro de Geografia e Estatística (IBGE) em adolescentes de 10 a 19 anos de idade, foi identificado excesso de peso nos dois sexos, em 16\% a 19\% nas Regiões Norte e Nordeste, com maior incidência nas Regiões Sudeste, Sul e Centro-Oeste de 20\% a 27\%.

Estudos da Organização Mundial de Saúde (OMS, 2009) desenvolvidos na região Europeia em 36 países, com crianças e adolescentes entre II e I 3 anos em 2005/2006, indicam prevalência de sobrepeso entre 5\% a 25\% e, quando comparados com dados de 200 I/2002, indicam que esta situação não diminuiu desde 2001 , exceto em apenas quatro países, tanto para meninos quanto para meninas.

O estudo realizado por Giugliano e Carneiro (2004) envolvendo 452 estudantes de escolas privadas de Taguatinga - DF, na faixa etária dos 6 aos 10 anos, detectou índices de sobrepeso e de obesidade da ordem de 16,8\% e 5,3\%, respectivamente.

Dessa forma, a obesidade se apresenta como um dos principais problemas de saúde pública da sociedade moderna, levando em conta seu caráter epidemiológico e suas consequências para a saúde da população em todas as faixas etárias, como resultado das transições demográficas, epidemiológicas e nutricionais ocorridas nas últimas décadas, que proporcionaram grandes alterações no estilo de vida das pessoas (LIMA et al., 2004; RINALDI et al., 2008).

Considerando a gravidade do problema e o seu acentuado crescimento, McDonald (2007) ressalta a necessidade de se incentivar a realização de pesquisas focadas na obesidade e nas suas consequências para a saúde.

Vale lembrar que uma das principais causas da obesidade é o sedentarismo, cujo controle demanda a implementação de programas que visem à promoção de um "estilo de vida" mais ativo, considerado como um fator fundamental para a promoção da saúde (NAHAS, 2003; FLORIANI; KENNEDY, 2007; PATE et al., 201 I ).

$\mathrm{Na}$ atualidade, uma criança gasta, em média, $600 \mathrm{kcal}$ diárias a menos do que há 50 anos e dedica 27 horas semanais do seu tempo a assistir à TV, o que constitui sua principal atividade (ALVES, 2003). Nesse sentido, Guinhouya (2007) chama a atenção para o fato de a TV compor um estilo de vida sedentário que, 
associado ao hábito de se alimentar em frente à televisão, desequilibra o balanço energético e se reflete na elevação dos índices de obesidade com todos os riscos para a saúde, onde se sobressaem as doenças cardiovasculares, o diabetes melitus e as dislipidemias (SANTOS et al., 2008; GUIDI et al., 2008).

A escola pode assumir um papel importante na promoção da saúde de crianças e adolescentes por ser um local onde essa população passa a maior parte do seu tempo, ocupada com experiências motoras e intelectuais, que, além de contribuírem para a construção da identidade e da auto-imagem, podem favorecer a promoção de um estilo de vida saudável (BRACCO et al., 2003). Essa importância foi confirmada nos estudos realizados por Veugelers e Fitzgerald (2005), com a implantação de um programa alimentar e de exercício físico escolar, o qual foi efetivo para prevenir obesidade infantil.

O levantamento de informações referentes à proporção de crianças com excesso de peso e obesidade representa uma medida importante, já que os estudos existentes no Brasil, além de escassos, são divergentes.

Neste contexto, este estudo pretendeu contribuir para a superação da escassez de dados relacionados à obesidade no Brasil - especificamente na Região Centro-Oeste - tendo como objetivo traçar um perfil representativo do excesso de peso, da atividade física regular e dos hábitos alimentares, em estudantes de escolas públicas e privadas da cidade de Anapólis-GO.

\section{MATERIAL E MÉTODOS}

O estudo assumiu um caráter epidemiológico, com delineamento transversal, envolvendo crianças de 7 a 9 anos, de ambos os sexos, matriculadas em escolas públicas e privadas da cidade de Anápolis-GO.

A amostra foi obtida de forma estratificada, dividindo-se a cidade em cinco regiões (centro, norte, sul, leste e oeste). Para cada região, foram selecionadas as duas maiores escolas - uma pública e uma privada - totalizando I 0 escolas e 1982 estudantes, conforme caracterização apresentada no Quadro I . 
Quadro I. Caracterização da amostra.

\begin{tabular}{|c|c|c|c|c|c|c|c|c|}
\hline \multirow{3}{*}{ Região } & \multicolumn{3}{|c|}{ Escolas } & \multicolumn{4}{|c|}{ Número de alunos } & \multirow{3}{*}{ Total } \\
\hline & \multirow{2}{*}{ Pública } & \multirow{2}{*}{ Privada } & \multirow[t]{2}{*}{ Total } & \multicolumn{2}{|c|}{ Pública } & \multicolumn{2}{|c|}{ Privada } & \\
\hline & & & & Fem. & Masc. & Fem. & Masc. & \\
\hline Centro & I & 1 & 2 & & & & & \\
\hline Norte & I & । & 2 & & & & & \\
\hline Sul & । & I & 2 & 607 & 653 & 361 & 361 & 1987 \\
\hline Leste & I & 1 & 2 & & & & & 1702 \\
\hline Oeste & I & I & 2 & & & & & \\
\hline Total & 5 & 5 & 10 & & 60 & & & \\
\hline
\end{tabular}

\section{INSTRUMENTOS}

Para a realização do estudo, recorreu-se a dois procedimentos distintos e complementares:

I) Utilizaram-se as Medidas Antropométricas (peso corporal e estatura) para cálculo do Índice de Massa Corporal (IMC), empregado para classificar as crianças em obesas e sobrepesadas, segundo pontos de cortes definidos por Cole et al. (2000), que levam em conta, além dos valores do IMC, o sexo e a idade da criança.

II) Para avaliar o nível de atividade física e os hábitos alimentares, foi utilizada uma versão adaptada do questionário proposto por Barrros et al. (2003) que, além de analisar os hábitos alimentares, serviu para identificar um dia típico de atividades físicas (DAFA), levantando o tipo, a quantidade e a intensidade das atividades mais praticadas, considerando o lazer, as atividades escolares e os deslocamentos para a escola e os trabalhos domésticos.

Quanto ao cálculo das atividades físicas, o questionário permite levantar, por meio de informações retrospectivas de auto-recordação, informações sobre os aspectos demográficos (sexo e idade), a percepção de atividade física, a distância de casa até a escola e o meio de transporte utilizado, as atividades esportivas, as tarefas domésticas e as atividades de lazer do avaliado.

Para a conversão das informações relativas à atividade física em valores estimados de dispêndio energético, e a consequente transformação dessas informações em escores matemáticos, recorreu-se ao compêndio de atividades físicas proposto por Ainsworth et al.(2000), que oferecem informações sobre o gasto energético, em unidades do equivalente metabólico (MET) de trabalho para uma atividade particular.

Após a apuração e cálculo isolado de cada atividade, foi realizado o cálculo do Escore Geral (EG) de cada aluno, pela somatória e multiplicação dos fatores relativos ao tipo de atividade física, à intensidade do esforço (leve, moderado, vigoroso) e à frequência semanal dessas atividades, com base na equação abaixo: 
Escore Geral $(E G)=[($ transporte*distância $) * 2 * 5)+($ dança*frequência semanal $)+($ futebol*frequência semanal $)+($ ciclismo*frequência semanal $)+($ tarefas domésticas*frequência semanal) + (lazer $\mid$ *frequência semanal) + (lazer2*frequência semanal) + (lazer $3 *$ frequência semanal)] .

Na sequência, foram estabelecidos os pontos de corte, relativamente ao EG obtido (soma das atividades físicas) usando a classificação dos quartis. Foram consideradas inativas as crianças com escore abaixo do percentil 25, insuficientemente ativas as de percentil entre 25 e 50, moderadamente ativas as que apresentaram percentil entre 50 e 75 e ativas, aquelas com valores acima do percentil 75.

O mesmo instrumento (questionário) serviu, ainda, para avaliar os hábitos alimentares, considerando cinco refeições de um dia típico: café, lanche, almoço, lanche e jantar.

Para o cálculo dos hábitos alimentares, utilizou-se como referência a nova pirâmide alimentar (BARBOSA; COSTA; SOARES, 2006) para classificar os nutrientes e as porções dos alimentos, segundo orientações estabelecidas no Quadro 2.

Quadro 2. nutrientes e porções sugeridos pela nova Pirâmide Alimentar.

\begin{tabular}{lll}
\hline PORÇÕES & ALIMENTOS & NUTRIENTES \\
\hline I a 2 & Doce, refrigerante, mel, sorvete & Açúcares \\
5 a 9 & $\begin{array}{l}\text { Cachorro- quente, salgado, pão, cuscuz, biscoito, pizza, sopa, min- } \\
\text { gau, mandioca, inhame, sopa, batata e angu, arroz, soja e macarrão. }\end{array}$ & Carboidratos \\
3 & Carne, frango, ovos, peixe, feijão. & Proteínas \\
I a 2 & Maionese, batata frita, pizza. & Lipídeos \\
3 a 5 & Frutas. & Fibras, vitaminas e \\
\hline
\end{tabular}

Somadas as porções dos alimentos, foi realizada a classificação dos hábitos alimentares em adequado, insuficiente e excessivo, conforme definições apresentadas no Quadro 3.

Quadro 3. Classificação dos Hábitos Alimentares.

CLASSIFICAÇÃO DOS HÁBITOS ALIMENTARES

Nutrientes dentro das porções recomendadas. Foi considerada como alimentação adequada.

Nutrientes abaixo das porções recomendadas. Foi considerada como alimentação insuficiente.

Nutrientes acima das porções recomendadas. Foi considerada como alimentação excessiva.

O projeto foi submetido à apreciação do Comitê de Ética em Pesquisa da Universidade Federal da Paraíba, e aprovado sob parecer RG 129/05, referendado pelo Comitê de Ética da Universidade Católica de Brasília, conforme Resolução 196/96 do Conselho Nacional de Saúde. 
Para o tratamento dos dados, foi realizada, primeiramente, uma análise descritiva dos dados e o cálculo de percentis para classificação do nível de atividade física. Em seguida, utilizou-se o teste "t" de Student para amostras independentes e para a análise de variância (One-Way ANOVA), para verificar diferenças entre as variáveis analisadas.

\section{RESULTADOS}

A Tabela I apresenta os resultados de sobrepeso e obesidade dos escolares masculinos e femininos, segundo o tipo de escola (pública ou privada), bem como a idade e o sexo das crianças integrantes da amostra. Os dados revelam, para as escolas públicas, um total de 14,35\% de estudantes com excesso de peso, enquanto nas escolas privadas esse índice foi superior a 25\%. Esses resultados tornam-se preocupantes, uma vez que as crianças com idades mais elevadas apresentam índices de excesso de peso, também, mais elevados.

Tabela I. IMC dos meninos e meninas com excesso de peso em escolas públicas e privadas.

\begin{tabular}{|c|c|c|c|c|c|c|c|c|c|c|}
\hline \multirow{2}{*}{ Idade } & \multirow{2}{*}{ Sexo } & \multicolumn{2}{|l|}{$N$} & \multirow{2}{*}{$\begin{array}{c}\text { Total N } \\
\text { Geral }\end{array}$} & \multicolumn{2}{|c|}{ \% sobrepeso } & \multicolumn{2}{|c|}{ \% obesidade } & \multicolumn{2}{|c|}{$\begin{array}{l}\text { Total geral \% } \\
\text { sobrepeso e } \\
\text { obesidade }\end{array}$} \\
\hline & & *Public. & ***Priv. & & *Public. & ***Priv. & *Public. & ***iv. & *Public. & *** Priv. \\
\hline \multirow{2}{*}{6,5} & M & 22 & 10 & 32 & 18,18 & 20 & 4,54 & 20 & 22,72 & 40 \\
\hline & $F$ & 22 & 05 & 27 & 9,09 & 40 & 4,54 & 00 & 13,63 & 40 \\
\hline \multirow{2}{*}{7} & M & 72 & 37 & 109 & 8,33 & $|3,5|$ & 2,77 & 5,41 & 11,10 & 18,92 \\
\hline & $F$ & 68 & 32 & 100 & 13,23 & 18,75 & 2,94 & 21,88 & 16,77 & 40,63 \\
\hline \multirow{2}{*}{7,5} & M & 142 & 47 & 189 & 9,15 & 8,51 & 5,63 & 8,51 & 14,78 & 17,02 \\
\hline & $\mathrm{F}$ & 121 & 46 & 167 & 6,61 & 21,74 & 7,43 & 6,52 & |4,04 & 28,26 \\
\hline \multirow{2}{*}{8} & M & 142 & 70 & 212 & 10,56 & 18,57 & 4,22 & 2,86 & 14,78 & 21,43 \\
\hline & $\mathrm{F}$ & 135 & 63 & 198 & 8,88 & 15,87 & 0,74 & I, I I & 9,62 & 16,98 \\
\hline \multirow{2}{*}{8,5} & M & 135 & 78 & 213 & 13,23 & 20,51 & 3,70 & 2,56 & 17,03 & 23,07 \\
\hline & $F$ & 122 & 74 & 196 & 6,55 & 29,73 & 3,27 & 4,05 & 9,82 & 13,78 \\
\hline \multirow{2}{*}{9} & M & 118 & 72 & 190 & 11,86 & 18,06 & 5,08 & 6,94 & 16,94 & 25,00 \\
\hline & $F$ & 122 & 99 & 221 & 11,47 & 17,17 & 4,09 & 4,04 & 15,56 & 21,21 \\
\hline \multirow{2}{*}{9,5} & $M$ & 22 & 47 & 69 & 18,18 & 10,64 & 4,54 & 8,51 & 22,72 & 19,15 \\
\hline & $F$ & 17 & 42 & 59 & 17,64 & 21,43 & 0,00 & 7,14 & 17,64 & 28,57 \\
\hline Total & & 1260 & 722 & 1982 & $|0,3|$ & 18,55 & 4,04 & 6,65 & 14,35 & 25,02 \\
\hline
\end{tabular}


A classificação dos hábitos alimentares é apresentada na Tabela 2, onde se verifica que a maioria dos alunos $(84,8 \%)$ encontra-se com uma alimentação considerada insuficiente, e apenas 1 । ,6\% dos escolares apresentaram hábitos alimentares adequados, segundo os referenciais da nova pirâmide alimentar.

Tabela 2. Classificação dos escolares segundo seus hábitos alimentares.

\begin{tabular}{lll}
\hline Classificação & Frequência & Porcentagem \\
\hline Adequada & 229 & 11,6 \\
Insuficiente & $\mathbf{1 6 8 2}$ & $\mathbf{8 4 , 8}$ \\
Inadequada & 71 & 3,6 \\
Total & 1982 & 100,0 \\
\hline
\end{tabular}

Em relação ao nível de atividade física dos escolares, o Gráfico I destaca que a maioria (quase 60\%) dos alunos da rede privada foi considerada inativa ou insuficientemente ativa. $\bigcirc$ quadro muda um pouco em relação aos estudantes da rede pública, onde $43 \%$ dos estudantes são considerados inativos ou insuficientemente ativos, o que revela uma situação vantajosa para os alunos da rede pública, relativamente à prática regular de atividades físicas. Constatou-se, também, que os meninos de ambas as redes escolares são significativamente mais ativos que as meninas $(p \leq 0,000)$.

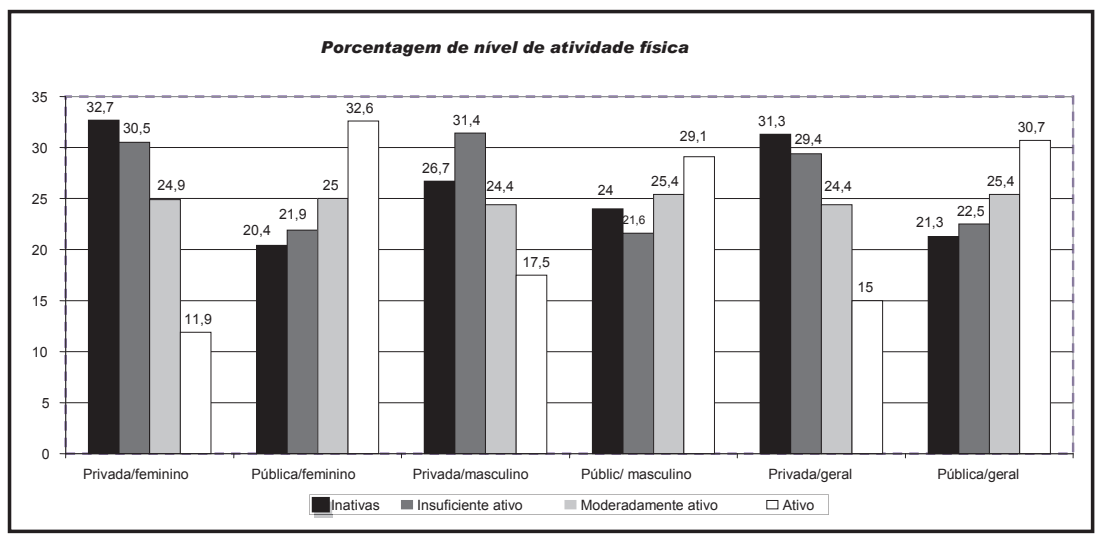

Gráfico I. Nível de atividade física dos escolares, por sexo e natureza da escola. 


\section{DISCUSSÃO}

Os dados encontrados no estudo confirmam a tendência mundial para elevação acentuada do número de obesos, a ponto de a obesidade ser considerada uma epidemia (SAVOYE et al., 2007; RINALDI et al., 2008; ORSI; HALE; LYNCH, $20 \mathrm{I}$ ). Ficou constatado que as crianças da rede privada de ensino apresentam níveis de excesso de peso mais elevados que as crianças da rede pública. $\bigcirc$ estudo desenvolvido por Utecht, Soares e Serassuelo Junior (2008) confirma que os jovens com maior poder aquisitivo são os que mais sofrem com obesidade e sobrepeso, sem negar que o problema aflige, também, os estudantes de escolas públicas, onde, predominantemente, estudariam as crianças e jovens oriundos das classes sociais menos favorecidas. No que concerne à diferenciação entre meninos e meninas, foi constatada, neste estudo, a mesma tendência encontrada nos estudos realizados por Silva, Balaban e Motta, (2005) e Santos et al. (2008), que é a predominância do excesso de peso entre os alunos do sexo masculino. Sendo assim, indispensável que se inicie a prática de exercício físico para a melhora da resposta inflamatória de obesos (PRADO et al., 2009).

Este estudo constatou, também, que os meninos são significativamente $(p \leq 0,000)$ mais ativos que as meninas, embora os níveis de atividade física, para ambos os sexos, se revelem baixos ou insuficientes. Os meninos brincam mais e praticam esportes, enquanto as meninas ajudam mais nas tarefas domésticas e raramente praticam esportes ou participam de brincadeiras mais ativas. Esses dados reforçam os resultados encontrados no estudo de Pereira e Barros (2004), onde também os meninos mostraram-se mais ativos do que as meninas.

Segundo Floriani e Kennedy (2007), o problema do baixo nível de atividade física tende a atingir crianças do mundo inteiro. Teixeira et al. (2005) e Tammelin et al. (2007) ressaltam a necessidade de aumentar os níveis dessa atividade entre os jovens, bem como de reverter seus hábitos comportamentais sedentários como, por exemplo, o tempo excessivo gasto na frente da televisão.

$O$ problema se acentua e assume gravidade maior na medida em que os hábitos alimentares incorretos acompanham a tendência ao sedentarismo. Enquanto em um passado ainda próximo, as preocupações nutricionais focavam-se nos problemas resultantes da pobreza e da consequente subnutrição, hoje há uma crescente preocupação com o sobrepeso e a obesidade, que é crescente, mesmo entre as classes menos favorecidas.

Os dados levantados neste estudo demonstraram que os escolares, tanto das escolas públicas quanto das privadas, não se alimentam de forma adequada. A maioria faz uso de uma alimentação considerada insuficiente, segundo o referencial 
estabelecido pela nova pirâmide alimentar (BARBOSA, COSTA, SOARES, 2006), necessitando, assim, de estudos que promovam uma mudança no quadro de sedentarismo e má alimentação, como pode ser visto no estudo de Dâmaso et al. (2006) com 28 adolescentes que participaram de um tratamento multidisciplinar não medicamentoso (atividade física, alimentação balanceada e apoio psicológico) por um período de 12 semanas, que encontrou resultados eficientes ao reduzir adiposidade visceral e promover alterações desejáveis no perfil de grelina e leptina.

Triches e Giugliani (2005), ao avaliarem a associação da obesidade com as práticas alimentares e o conhecimento de nutrição, constataram que a obesidade mostrou-se associada ao maior nível de conhecimento sobre nutrição e à prática de hábitos alimentares pouco saudáveis. Isso foi constatado no presente estudo pelo maior percentual de obesidade encontrado entre os escolares da rede privada, onde predominam hábitos alimentares pouco saudáveis, em que pese o fato de haver, presumivelmente, um maior nível de conhecimento sobre o assunto.

O estudo de Cano et al. (2005), realizado em duas escolas (pública e privada) da cidade de Franca-SP, com crianças de 7 a 8 anos, detectou o problema da obesidade associado aos alimentos industrializados e calóricos que eram vendidos nas cantinas das escolas.

Da mesma forma, os resultados obtidos no estudo de Mascarenhas e Santos (2006) e de Teixeira et al. (20 l 0) indicam que os cardápios oferecidos pelas escolas públicas não atendem aos objetivos nutricionais propostos pelo Programa Nacional de Alimentação Escolar (PNAE). Gonzalez (2007) Jomaa, MCdonnell e Probart (20। I) ressaltam a importância da implementação de uma educação nutricional como elemento essencial à saúde e ao bem-estar da população, contribuindo para modificar ou prevenir hábitos que, se não forem corrigidos precocemente, poderão se agravar com resultados desastrosos, onde se sobressairão as consequências negativas sobre a saúde, produzidas pelo excesso de peso corporal.

\section{CONCLUSÃO}

Os resultados encontrados revelaram que os escolares da amostra apresentam um nível elevado de sobrepeso e obesidade, com maior predominância dos alunos da rede de ensino privada, frente aos estudantes das escolas públicas.

Quanto ao nível de atividade física, foi constatado que os escolares da rede pública de ensino são mais ativos que os da rede privada, e que ambos despendem uma parte significativa de seu tempo livre assistindo à televisão.

Quanto aos hábitos alimentares, podemos concluir que os escolares estudados nesta pesquisa adotam hábitos insuficientes em relação ao que é sugerido pela nova pirâmide alimentar. 
Por fim, acreditando nos resultados encontrados no estudo, esperamos que venham a surgir propostas e programas por parte dos governos, das escolas e das organizações sociais e familiares, destinando um espaço maior ao profissional de saúde para o desenvolvimento de atividades profiláticas, voltadas à conscientização acerca dos benefícios de um estilo de vida ativo (atividade física e hábitos alimentares) para as crianças, a fim de que tenham, no presente e no futuro, uma vida mais saudável.

\section{Overweight, Level of Physical Activity and Food Habits at School in the City of Anápolis-GO}

ABSTRACT: This study aimed to identify excess of weight, level of physical activity and eating habits among students aged between 7 and 9 years. The study was of epidemiological character, cross-sectional, involving 1982 children. Results showed that overweight was lower among students of public schools. Regarding the level of physical activity, it was found that the majority of students were considered sedentary or irregularly active, and that public school students are more active. It was concluded that the students adopt levels of physical activity and eating habits far below of what guidelines suggest by the scientific literature. KEYWORDS: Overweight; usual physical activity; dietary habits; students.

\section{Exceso de peso, nivel de actividad física y hábitos alimentarios en escolares de la ciudad de Anápolis-GO}

RESUMEN: Este estudio tuvo como objetivo identificar el exceso de peso, el nivel de actividad física y los hábitos alimentarios, en escolares con edad entre 7 y 9 años. El estudio fue de carácter epidemiológico, con delineamiento transversal, envolviendo 1982 niños. Los resultados demuestran que el exceso de peso fue menor en los estudiantes de la enseñanza pública. En relación al nivel de actividad física, se comprobó que la mayoría de los escolares fue considerada sedentaria o con actividad física insuficiente, y que los estudiantes de las escuelas públicas son más activos. Se llegó a la conclusión que los escolares adoptan el nivel de actividad física y hábitos alimentarios insuficientes en relación a las orientaciones sugeridas por la literatura científica.

PALABRAS CLAVES: Exceso de peso; actividad física habitual; hábitos alimentícios; escolares.

\section{REFERÊNCIAS}

AINSWORTH, B. E. et al. Compendium of physical activities: an update of activity codes and met intesities. Medicine and Science in Sports and Exercise, v. 32, p. S498-504, Sep. 2000. Suppl. 9. 
ALVES, J. G. B. Atividade física em crianças: promovendo a saúde do adulto. Revista Brasileira de Saúde Materno Infantil, Recife, v. 3, n. I, p. 5-6, jan./mar. 2003.

BANKOFF, A. D. P.; MOUTINHO, E. A. Obesidade infantil e avaliação em pré-escolares. Revista Brasileira de Ciências do Esporte, Campinas, v. 23, n. 3, p. I05-120, maio. 2002.

BARBOSA, R. M. S; COSTA, R. S; SOARES, E. A. Guias alimentares para crianças: aspectos históricos e evolução. Revista de Nutrição, Campinas, v. 19, n. 2, p. 255-263, mar/abr. 2006.

BARROS, M. V. G. et al. Desenvolvimento e validação do questionário dia típico de atividades físicas e de alimentação. In: BARROS, M. V. G.; NAHAS, M. V. Medidas da atividade física: teoria e aplicação em diversos grupos populacionais. Londrina: Midiograf; 2003.

BRACCO, M. M. et al. Atividade física na infância e adolescência: na saúde pública. Revista de Ciências Médicas, Campinas, v. 12 n. I , p. 89-97, 2003.

CANO, M. A. T. et al. Estudo do estado nutricional de crianças na idade escolar na cidade de Franca- SP: Uma introdução ao problema. Revista Eletrônica de Enfermagem, Goiânia, v. 7, n. 2, p. 179-184, 2005. Disponível em: <http://www.fen.ufg.br> Acesso em: 12 out. 2006.

COLE, T. J. et al. Establishing a standard definition for child overweight and obesity worldwide: international survey. BMJ-British Medical Journal, v. 320, p. 1240-1243, may 2000. Disponível em: < http://www.pubmedcentral.nih.gov/articlerender.fcgi?artid=27365> . Acesso em: 15 out. 2004.

DAMASO, A. R. et al. Tratamento multidisciplinar reduz o tecido adiposo visceral, leptina, grelina e a prevalencia de esteatose hepática não alcoólica (NAFLD) em adolescentes obesos. Revista Brasileira de Medicina do Esporte. v. I2, n. 5, set./out, 2006.

FLORIANI, V.; KENNEDY, C. Promotion of physical activity in primary care for obesity treatment/prevention in children. Current Opinion in Pediatrics, v. 19, n. I, p. 99- 103, Feb. 2007.

GIUGLIANO R; CARNEIRO E C. Factors associated with obesity in school children. Jornal de Pediatria, Rio de Janeiro, v. 80, p. 17-22, 2004.

GUINHOUYA, C.B. Physical activity and sedentary lifestyle in children as time-limited functions: Usefulness of the principal component analysis method. Behavior Research Methods, v. 39, n. 3, p. 682-688, aug. 2007.

GONZALEZ, J. School-based Nutrition and Healthy Lifestyle Obesity Prevention Program Shows Success. Journal Nute Education and Behavior, v. 39, n. 4, jul./aug. 2007. Supp. I.

GUIDI, R. M. et al. Incidência da obesidade e sobrepeso em escolares da rede pública de ensino da cidade de Campinas/SP. In: CONGRESSO BRASILEIRO DE METABOLISMO. NUTRIÇÃO E EXERCíCIO, 2., Londrina, 2008. Anais do ... Londrina, 2008. P. I I 5- I I 8.

INSTITUTO BRASILEIRO DE GEOGRAFIA E ESTATÍ́STICA (IBGE), 2008/2009. Disponível em http://www.ibge.gov.br/home/opulaçãoa/opulação/condicaodevida/pof/2008_2009_encaa/comentario.pdf. Acesso em: 25 out. 2011 . 
JOMAA, L. H; MCDONNELL, E.; PROBART,C. School feeding programs in developing countries: impacts on children's health and educational outcomes. Nutrition Reviews, v. 69, n. 2, p. 83-98, feb. 2011 .

LIMA, S. C. V. C. et al. Perfil lipídico e peroxidação de lipídeos no plasma em crianças e adolescentes com sobrepeso e obesidade. Jornal de Pediatria, Porto Alegre, v. 80, n. I, p. 23-28, 2004.

MASCARENHAS, J. M. O; SANTOS, J. C. Avaliação da composição nutricional dos cardápios e custos da alimentação escolar da rede municipal de Conceição do Jacuípe. Feira de Santana. Revista Sitientibus, Feira de Santana, n. 35, p.75-90, jul./dez. 2006.

McDONALD, S. D. Management and prevention of obesity in adults and children. Canadian Medical Association Journal, v. 176, n. 8, p.। 109-I I 10, abr. 2007. Disponível em:<http:// www.ecmaj.com/cgi/content/full/I76/8/I I09>. Acesso em: 10 sept. 2010.

NAHAS, M.V. Atividade física, saúde e qualidade e vida. 3. ed. Londrina: Midiograf, 2003. 278p.

OLIVEIRA, C. L.; FISBERG, M. Obesidade na infância e adolescência: - uma verdadeira epidemia. Arquivo Brasileiro de Endocrinologia e Metabolismo, São Paulo, v. 47, n. 2, p. 107 108, abr. 2003.

ORGANIZAÇÃO MUNDIAL DE SAÚDE (OMS). Prevalence of overweight and obesity in children and adoles-cents. FACT SHEET 2.3, CODE: RPG2_Hous_E2,December 2009. Disponível em: http://www.euro.who.int/__data/assets/pdf_file/0005/96980/2.3.-Prevalence-of-overweight-and-obesity-EDITED_layouted_V3.pdf. Acesso em: 25.oct. 20 I I.

ORSI, C. M.; HALE, D. E; LYNCH, J. L. Pediatric obesity epidemiology. Current Opinion in Endocrinology, Diabetes \& Obesity, v. |8, n. I, p. |4-22, 201 I.

PATE, R. R. et al. Overcoming barriers to physical activity: helping youth be more active. ACSM'S Health \& Fitness Journal, v. I5, n. I, p. 7-12, jan./fev. 201।.

PEREIRA, R. R.; BARROS, J. F. Estilo de vida dos escolares de Montes Claros, MG. Revista Digital, Buenos Aires, ano 10, n. 75, ago. 2004. Disponível em: <http://www.efdeportes. com/efd75/escolar.htm>. Acesso em: 22 mar. 2010.

PRADO, W. L. do. Obesidade e adipocinas inflamatórias: implicações práticas para a prescrição de exercício. Revista Brasileira de Medicina do Esporte. v. I5, n. 5, set/out, 2009.

RINALDI, A. E. M. et al. Contribuições das práticas alimentares e inatividade física para o excesso de peso infantil. Revista Paulista de Pediatria, São Paulo, v. 26, n. 3, p. 27 I-277, 2008.

SANTOS, M. G. dos et al. Fatores de risco no desenvolvimento da aterosclerose na infância e adolescência. Arquivo Brasileiro de Cardiologia, São Paulo, v. 90, n. 4, p. 30 I-308, abr. 2008.

SILVA, G. A.; BALABAN, G.; MOTTA, M. E. F. Prevalência de sobrepeso e obesidade em crianças e adolescentes de diferentes condições socioeconômicas. Revista Brasileira de Saúde Materno Infantil, Recife, v. 5, n. I, p. 53- 59, jan. 2005. 
SAVOYE, M. et al. Effects of a Weight Management Program on Body Composition and Metabolic Parameters in Overweight Children: a randomized controlled trial. Journal of the American Medical Association, v. 297, n. 24, p. 2697-704, Jun. 2007.

TAMMELIN, T. et al. Physical activity and sedentary behaviors among finnish youth. Medicine \& Science in Sports \& Exercise, v. 39, n. 7, p. 1067-1074, July. 2007.

TEIXEIRA, C. G. O. et al. Nível de atividade física nos períodos de aula e de férias, em escolares de Anápolis-GO. Revista Brasileira de Ciência e Movimento, Brasília, v. I 3, n. I, p. 45-49, 2005.

TEIXEIRA, C. G. O. et al. Análise quanto ao valor calórico dos lanches oferecidos nas cantinas das escolas. Coleção Pesquisa em Educação Física, Jundiaí, v. 9, n. 3, p. 39-44, 2010.

TRICHES, R. M.; GIUGLIANI, E. R. J. Obesidade, práticas alimentares e conhecimentos de nutrição em escolares. Revista de Saúde Pública. São Paulo, v. 39, n. 4, p. 54I-7, ago. 2005.

UTECHT, E. C.; SOARES, L. E. P.; SERASSUELO JUNIOR, H. Prevalência de sobrepeso e obesidade em escolares pertencentes ao ensino público da região Central do município de Cambe/PR. In: Congresso Brasileiro de Metabolismo. Nutrição e Exercício, Londrina, 2008. Anais do ... Londrina, 2008. P. 212.

VEUGELERS, P. J.; FITZGERALD, A. L. Effectiveness of school programs in preventing childhood obesity: a multilevel comparison. American Journal of Public Health, v.95, n.3, p. 432-435, mar. 2005.

Recebido em: 22 fev. 2011 Aprovado em: 3 out. 2011

Endereço para correspondência: Patrícia Espíndola Mota Venâncio Rua Leopoldo de Bulhões, no I0। 4. Bairro Maracanã Anápolis-G0 CEP: 75040-500 